\title{
HIPERTRANS: High Performance Transport Network Modelling and Simulation
}

\author{
Stephen E. Ijaha, Stephen C. Winter, and Nasser Kalantery \\ Centre for Parallel Computing, Cavendish School of Computer Science, \\ University of Westminster, 115 New Cavendish Street, London W1M 8JS, UK \\ \{ijahas, wintersc, kalantn\}@wmin.ac.uk \\ http://www.wmin.ac.uk
}

\begin{abstract}
HIPERTRANS (High Performance Transport network modelling and Simulation) is a fast and visually representative simulator that can predict traffic on a given urban road network. It was designed using object-oriented techniques and by re-engineering established road traffic models. It has the capability of interfacing to a range of UTC (urban traffic control) systems. Its high performance version was implemented by using a novel parallel programming platform called SPIDER; it has the capability of executing faster than real time and runs on distributed processors. The simulator provides a powerful GUI (graphical user interface) for entering road network models, configuring simulation runs, and visualising simulation results. The system provides helpful traffic diagnostic tools enabling local transport authorities, policy makers, researchers, and UTC manufacturers to gainfully exploit its functionality. The HIPERTRANS project was funded by the European Commission under the 4th Framework programme.
\end{abstract}

\section{Introduction}

\subsection{The HIPERTRANS Requirements and Specifications}

Traffic congestion problems in urban areas have prompted governments, local authorities and UTC manufacturers to be interested in a modelling and simulation tool that can be used to predict traffic situations. Such a tool is needed to achieve real-time traffic control measures and assist policy makers to make informed decisions [1]. The HIPERTRANS project conceived the idea of a fast, representative, flexible and visually comprehensive traffic simulation tool that can be achieved through the application of low cost but high performance computing environments, advanced simulation technologies, and industrial software production methodologies [2]-[3].

The HIPERTRANS simulation system consists of a new range of facilities for transport consultants, researchers, traffic engineers and UTC centre managers. The tool can enable transportation network operators to assess the performance of their road network quickly under a variety of operational conditions and behavioural patterns. Additionally, it can be used as a tool for hardware commissioning and operator training. Using event driven approach, the HIPERTRANS system is capable of putting vehicles into a network and representing the movement of individual vehicles. 
This paper describes the work carried out within HIPERTRANS during April 1997 to June 1999. After the description of the project in this Section, the next Section states the objectives of the work. Section 3 describes the methodology and approach employed by which these objectives were achieved. Section 4 provides the results achieve. The conclusions and recommendations for future research are presented in Section 5 .

\subsection{HIPERTRANS Partnership and Test Sites}

HIPERTRANS, an EC-funded consortium, was led by the University of Westminster (UoW) and had 8 partners spread across 4 different EC countries. The partners participated within two broad groups: Technology Group and User Group. The 3 Technology Group partners came from 2 universities - Facultés Universitaires NotreDame de la Paix à Namur (FUNDP) in Belgium and UoW in the UK; and a research institution - Institut National de Recherche en Informatique et Automatique (INRIA), in France. They were responsible for the definition, design, building, testing, and verification of the simulators that were developed within the project. The 5 User Group partners were formed by: 2 transport consultants - BKD Consultants Ltd (BKD), and W.S. Atkins (WSA), both in the UK, 2 UTC (urban traffic control) system manufacturers - Electronic Trafic S.A. (ETRA), in Spain and Peek Traffic Limited (PTL), UK, and a software house - SIMULOG in France. The User's main role was to validate and demonstrate the simulators in a real traffic environment using real traffic scenarios.

The work on the real-time simulator and predictor made enormous use of two different kinds of proprietary UTC systems kindly made available to the project by two of our partners: SCOOT [4] partly owned by PTL; and STU (Sistema de Trafico Urbano), [5] wholly owned by ETRA. SCOOT is the predominant type of UTC system in the UK and STU in Spain. The GUI developed for visualising the results and inputting road network data models into the simulator was developed by using Simulog's OGL graphical library. This library is based on the ILOG [6] system and is available on Windows and Unix platforms.

Two test sites, Hyde in the UK and Valencia in Spain were employed by the project. The criteria used in selecting these test sites were that they had the UTC systems used by our User partners and whose local authorities were willing to participate in the evaluation of the project. The participation of different UTC manufactures and local authorities in this project is also helping to overcome the challenges of noninteroperability of UTC systems not only in Europe but also throughout the world.

\section{Objectives}

The key aim of the project was to produce microscopic modelling and simulation tool for the easy and cost-effective development of road traffic control systems and for the effective management of traffic flow on road networks. Specific aims were to:

- develop a simulator able to interact with UTC systems at real-time speeds and develop a predictor consistent with users' requirement for look-ahead;

- implement a low-cost high-performance system on a scaleable workstation cluster; 
- demonstrate that the use of high-performance simulation can speed up and dramatically improve the operation of urban and inter urban transportation networks;

- compare the execution times performance of the simulation algorithms;

- model road traffic on a small-to-medium sized network; and study technology and best practice for extending the simulator to handle inter-urban situations.

In general, the HIPERTRANS system aimed to have the following basic functionality:

- performing microscopic simulation having a user-friendly graphical user interface;

- interfacing capability to UTC systems and capability of working in real time;

- being able to simulate and predict the state of the road traffic; and

- achieving high-performance computing on low cost platforms.

\section{Technical Description}

The implementation of the system was performed in three different but closely related stages involving the component software modules being developed on several sites and eventually integrated on a single platform. This provided essential co-ordination points within the software development and enabled the User Group to evaluate progress. They also assisted all partners to monitor and steer the development activities in an optimal manner. The strategy used in the work was geared towards applying results from the science base of advanced traffic modelling and control techniques as well as the technology of high-performance computing to achieve the simulators. The overall architecture consists of the simulator; a UTC system; and an interface between the simulator and the UTC system. The simulator is software and hardware configurable to:

- enable it to be used in real-time with different UTC types;

- provide a stand-alone simulation capability when not interfaced to a UTC;

- allow information relevant to a wide range of road networks to be used; and

- scale from low cost and moderate performance to higher cost higher performance.

The architecture shown in the Figure below is a combination of an object-oriented framework for traffic simulation, user simulation models, and application programming interfaces for interconnection to UTC systems. The background software used existed at three partner sites: PACSIM [7] developed by FUNDP; PROSIT [8] co-developed by INRIA and Simulog, and SPIDER [9] developed by UoW.

\begin{tabular}{|c|c|}
\hline \multicolumn{2}{|c|}{ GRAPHICAL USER INTERFACE } \\
\hline USER SIMULATION & \multirow{2}{*}{ SPIDER } \\
\hline PACSIM & \\
\cline { 1 - 1 } TRAFFIC SIMULATION FRAMEWORK & \\
\hline PROSIT & \\
\hline
\end{tabular}




\section{Results}

The project has delivered a micro-simulator as specified, and was used for highprofile public demonstrations and evaluation at Hyde in the UK and Valencia in Spain [10]. The results provide a proof of concept of high-performance simulation as ascertained from the needs of users. The resulting simulator can run on a network of workstations, ensuring that simulation of large traffic networks can be achieved within a pre-determined real time limit. Starting with the current road conditions supplied by an operator, the simulator was shown to run much faster than real time.

A testing exercise [10] was carried out to check the simulator's correctness, and to evaluate its performance by comparing the outputs from the sequential version with those from the parallel version. These measured the speed-up when different numbers of processors were employed compared to when only one processor was used. The correctness tests were designed to prove that the algorithms employed by both versions do not alter the nature of the results when the simulation is run under identical conditions. The strategies used in the 'correctness' test involved making the size of the road traffic model a variable parameter while keeping the number of vehicles generated by each of the two versions constant. In the performance test, the number of vehicles generated by each of the two versions was varied while keeping the size of the road traffic model constant. The results of the tests for one processor and using 4 processors show the different execution times for the two versions both using a grid road network and a total simulation time of 35 minutes for different number of vehicles. When the number of vehicles becomes very large, the speed up may approach a limit that is approximately near the theoretical limit $n$ where $n$ is the number of workstations used in the distributed version. For realistic as well as meaningful evaluation, the correctness tests were carried out on a real road network in Nice, south France.

\section{Summary and Conclusions}

Through the application of low cost but high performance computing environments, advanced simulation technologies and industrial software production methodologies within the project, a fast, representative, flexible and visually interactive traffic simulation tool have become available. This paper has described the work and results of the HIPERTRANS traffic simulation system. The software architecture employed comprises: the object-oriented framework for traffic simulation, PROSIT, PACSIM, and SPIDER. SIPIDER enabled the capability of distributed simulation so that the software resides on a cluster of workstations and provides a sequentially consistent parallel execution. The speed of the simulator permits faster than real-time prediction of road network behaviour to be made. The simulator can enable UTC operators to examine the future effect of their selected actions on levels of congestion fast enough to be able to revise and re-test the performance before selecting the best action to take.

The HIPERTRANS project has proven that microscopic simulation can be one of the most effective tools in the design and management of road traffic systems. In comparison to related work, other useful simulation tools [11]-[14] have been developed in the past, but further advancement in high performance simulation 
technology was motivated by the fact that large scale microscopic simulation are time consuming and so further research was carried out to look into the effective exploitation of distributed computing to speed up the simulation further in realistic road networks. This project has assured that the use of scalable parallel computing ensures that irrespective of the size and complexity of the modelled network, faster than real time criterion can be met.

Notwithstanding the advancement made within the HIPERTRANS project, more practical aspects need to be addressed. For future work, we recommend the development of an open simulation farmework which has the capability of integrating distributed executions with more user-friendly 3-dimensional graphical and animation interfaces, a wider variety of real-time UTC systems, and capability of receiving data from and sending data to traffic data and information systems. Future systems should not only be able to receive real-time traffic data and predict the future state of traffic, but should be able to incorporate emission models and GIS data. Other EC, national, and other research projects who have already built other types of simulators such as macroscopic simulators, emission models, and automatic debiting simulators, freight transport and crowd management simulators could benefit from HIPERTRANS results. It is possible for such other simulators to integrate with a microscopic road traffic simulator. Our collaboration with such builders will yield the possibilities of interfacing their work with our system. The purpose of such a modular and open simulator would be to use it in the area of transport, traffic and telematics where several opportunities are foreseen in typical applications such as traffic management, floating car data, and route guidance.

\section{Acknowledgement}

The HIPERTRANS project was funded by the European Commission's DG 7 under the Framework 4 programme. We are grateful to the EC and all the project partners.

\section{References}

1. HIPERTRANS Consortium, 1997, Deliverable D1: System Specification, HIPERTRANS RO-97-SC-1005, July 1997

2. IJAHA S.E. WINTER, S.C, KALENTERY N. and DANIELS B.K.: 'HIPERTRANS: a road traffic simulation as an operational tool', Paper No. 46 in the 1998 IEE International Conference on Simulations, University of York, UK, 30 Sept - 2 Oct 1998.

3. SIEGEL, G, FURMENTO, N, and MUSSI, P.: "A Traffic Simulator for Advance Transport Telematics (ATT) Strategies" IEEE conference Electrotechnological Services for Africa AFRICON'99, 29/09/99 - 01/10/99, Cape Town, South Africa.

4. PEEK Traffic Systems, 'SCOOT System Overview', 1995

5. ETRA I+D, 1996, STU-I+D-05-01, Version 3.1/1.2, Sistema de Trafico Urbano, Descripcion General STU.

6. HIPERTRANS Consortium: HIPERTRANS GUI and User's Manual, Annex 2 of Deliverable D5: Demonstration, HIPERTRANS RO-SC-1005, June 1999.

7. CORNELIS, E., and TOINT, P. L.: 'An introduction to PACSIM: A new dynamic behavioural model for traffic assignment', Report 1997/98.

8. GAUJAL, B., and MUSSI, P.: PROSIT Manual and HIPERTANS: Object Oriented Simulation for Urban Traffic', INRIA Internal Report, 1995. 
9. KALENTERY, N., SPIDER, Internal Report, Centre for Parallel Processing, University of Westminster, 1995

10. HIPERTRANS Consortium: Deliverable D5: HIPERTRANS Demonstration, HIPERTRANS RO-97-SC-1005, July 1999.

11. TSS: Transport Simulation Systems. AIMSUN. http://www.tss-ben.com/aimsun.html

12. Rickert M. and P. Wagner. Parallel Real-time Implementation of Large-scale, Route-plandriven Traffic Simulation. http://www.zpr.uni-koeln.de/GroupBachem/VERKEHR.PG/

13. PARAMICS: Traffic Simulation. PARAMICS, see also http://wwwusa.quadstone.com/paramics/ext/index.html

14. Nuttall I, and Fellendorf M.: VISSIM for Traffic Signal Optimisation. Traffic Technology International '96. Annual Review Issue. 1996. pp190-2. 